Real Analysis Exchange

Vol. 23(2), 1997-1998, pp. 813-816

Hussain Elalaoui-Talibi, Department of Mathematics, Huntington Hall \#10, Tuskegee University Tuskegee, AL 36088, e-mail: hussain@acd.tusk.edu and htal@mindspring.com

\title{
A REMARK ON GRAPHS OF MARCZEWSKI MEASURABLE FUNCTIONS
}

\begin{abstract}
We give a direct and simple proof of a result of Marczewski about graphs of $(s)$-measurable functions. We also prove some related general results about graphs of measurable functions in metric spaces from which Marczewski's result follows immediately.
\end{abstract}

\section{Introduction}

A subset $M$ of a complete separable metric space $X$ is Marczewski measurable if every perfect set $P$ in $X$ contains a perfect subset $Q$ such that either $Q \subseteq M$ or $Q \cap M=\emptyset$. We also say that $M$ is $(s)$-measurable or $M$ has property $(s)$. We also write $M \in s(X)$. The class of $(s)$-measurable sets of $X$ form a $\sigma$-algebra. A function $f: X \rightarrow Y$ where $Y$ is any metric space is Marczewski measurable or $(s)$-measurable if $f^{-1}(O) \in(s)$ for every open set $O \subseteq Y$. Marczewski has proved in [2] that a function $f: X \rightarrow Y$, where $X, Y$ are complete separable metric spaces, is $(s)$-measurable if and only if every perfect subset $P$ of $X$ contains a perfect set $Q$ such that the restriction of $f$ to $Q,\left.f\right|_{Q}$, is continuous. The latter property was introduced by Sierpinski in 1935.

Marczewski has stated in [2] that the graph of an $(s)$-measurable function $f: X \rightarrow Y$ is $(s)$-measurable in $X \times Y$, where $X, Y$ are complete separable metric spaces. He has not given a proof of this as well as some related results in that same paper. He mentioned that the proofs are similar to those used in the theory of functions in metric spaces, and he referred to [1] pp. 182, 183, 180 and a paper by him that was in preparation at the time. That paper was

\footnotetext{
Key Words: Marczewski measurable, perfect set, $\sigma$-algebra

Mathematical Reviews subject classification: Primary:28A20; Secondary: 26E99, $04 \mathrm{~A} 05$

Received by the editors December 5, 1997
} 
never published as far as we know, and what Marczewski had in mind was not quite clear.

We will first give a direct and simple proof to Marczewski's result. We will then prove some general results about measurable functions and their graphs in metric spaces from which Marczewski's result follows immediately.

\section{Graphs of $(s)$-measurable functions}

We give a direct and simple proof of Marczewski's result concerning graphs of (s)-measurable functions. We assume that $X$ and $Y$ are complete separable metric spaces.

Theorem 1. Let $f: X \rightarrow Y$ be $(s)$-measurable. Then its graph $G r(f)$ is (s)-measurable in $X \times Y$.

Proof. We have $G r(f)=\{(x, f(x)) \mid x \in X\}$. We need to show that every perfect set $P$ in $X \times Y$ contains a perfect set $Q$ such that either $Q \subseteq G r(f)$ or $Q \cap G r(f)=\emptyset$. So let $P \subseteq X \times Y$ be perfect. Consider the following two cases:

case1: $\prod_{X}(P)$, the projection of $P$ on $X$, is countable. So the set $P \backslash$ $\left\{(x, f(x)) \mid x \in \prod_{X}(P)\right\}$ is an uncountable $G_{\delta}$ set since its complement is countable. It must then contain a perfect set $Q$. Clearly $Q \cap \operatorname{Gr}(f)=\emptyset$.

case2: $\prod_{X}(P)$ is uncountable. Since this is the projection of a Borel set, it is analytic and it must contain a perfect set $P_{1}$. Since $f$ is $(s)$-measurable, there is a perfect set $P_{2} \subseteq P_{1}$ such that $\left.f\right|_{P_{2}}$ is continuous. Let $Q_{1}=\{(x, f(x)) \mid x \in$ $\left.P_{2}\right\} . Q_{1}$ is clearly perfect. If $P \cap Q_{1}=\emptyset$, then the set $Q_{2}=\{(x, y) \in P \mid x \in$ $\left.P_{2}\right\}$ is an uncountable closed set, and it must contain a perfect set $Q$. Clearly $Q \cap \operatorname{Gr}(f)=\emptyset$. If $P \cap Q_{1}$ is countable, then $P \backslash\left(P \cap Q_{1}\right)$ is uncountable, and it must contain a perfect set $Q$. Clearly $Q \cap G r(f)=\emptyset$. If $P \cap Q_{1}$ is uncountable, then it must contain a perfect set $Q$. Clearly $Q \subseteq G r(f)$. So $G r(f)$ is $(s)$-measurable which finishes the proof.

The converse of this Theorem is not true if we assume the continuum hypothesis. An example can be found in [2]. We still do not have any examples in (ZFC).

\section{Some General Results}

We will give some general results about measurable functions in metric spaces. We believe that these are the results that Marczewski had in mind about $(s)$-measurable functions and their graphs. If $X, Y$ are metric spaces with $\sigma$-algebras $\mathcal{F}$ and $\mathcal{G}$ respectively, the product $\sigma$-algebra on the product space 
$X \times Y$, denoted by $\mathcal{F} \otimes \mathcal{G}$ is the smallest $\sigma$-algebra that contains the measurable rectangles $A \times B$ where $A \in \mathcal{F}$ and $B \in \mathcal{G}$. If $\mathcal{F}_{1}, \mathcal{F}_{2}, \ldots$ are $\sigma$-algebras on the spaces $X_{1}, X_{2}, \ldots$, respectively, then the product sigma algebra $\mathcal{F}_{1} \otimes \mathcal{F}_{2} \otimes \ldots$ on $X_{1} \times X_{2} \times \ldots$ is the smallest $\sigma$-algebra that contains all the measurable cylinders $X_{1} \times \ldots \times X_{k-1} \times A \times X_{k+1} \times \ldots, \quad A \in \mathcal{F}_{k}, k=1,2, \ldots$ If a set $A$ is $\mathcal{F}$-measurable, we will write $A \in \mathcal{F}$. A function $f: X \rightarrow Y$ is $\mathcal{F}$-measurable if $f^{-1}(O) \in \mathcal{F}$ for every open set $O \subseteq Y$.

Lemma 2. Let $X, Y_{1}, Y_{2}, \ldots$ be metric spaces with $Y_{1}, Y_{2}, \ldots$ separable, and let $\mathcal{S}$ be a $\sigma$-algebra on $X$. Let $f_{i}: X \rightarrow Y_{i}, i=1,2, \ldots$. Define a function $F: X \rightarrow Y_{1} \times Y_{2} \times \ldots$ by $F=\left(f_{1}, f_{2}, \ldots\right)$. Then $F$ is $\mathcal{S}$-measurable if and only if $f_{i}, i=1,2, \ldots$ are $\mathcal{S}$-measurable.

Lemma 3. Let $X_{1}, X_{2}, \ldots$ be metric spaces with $\sigma$-algebras $\mathcal{S}_{1}, \mathcal{S}_{2}, \ldots$ respectively. Let $Y_{1}, Y_{2}, \ldots$ be separable metric spaces, and let $f_{i}: X_{i} \rightarrow Y_{i}, i=$ $1,2, \ldots$ be $\mathcal{S}_{i}$-measurable functions, respectively. Let $g: Y_{1} \times Y_{2} \times \ldots \rightarrow \mathbb{R}$ be a continuous function. Define the function $h: X_{1} \times X_{2} \times \ldots \rightarrow \mathbb{R}$ by $h\left(x_{1}, x_{2}, \ldots\right)=g\left(f_{1}\left(x_{1}\right), f_{2}\left(x_{2}\right), \ldots\right)$. Then $h$ is $\mathcal{S}_{1} \otimes \mathcal{S}_{2} \otimes \ldots$-measurable.

The proofs of these lemmas are similar to those of the results in [1] pp.182, 183.

Theorem 4. Let $X, Y$ be metric spaces with $\sigma$-algebras $\mathcal{S}$ on $X$, and $\mathcal{T}$ on $Y$. Assume that $Y$ is separable and that $\mathcal{T}$ contains the open sets of $Y$. Let $f$ be an $\mathcal{S}$-measurable function from $X$ to $Y$. Then the graph of $f, \operatorname{Gr}(f)$, is $\mathcal{S} \otimes \mathcal{T}$-measurable.

Proof. Note that $\operatorname{Gr}(f)=\{(x, y) \in X \times Y: \phi(x, y)=0\}$ where $\phi$ is the function from $X \times Y \rightarrow \mathbb{R}$ defined by $\phi(x, y)=d(y, f(x))$ where $d$ is the metric on $Y$. By the previous two lemmas $\phi$ is $\mathcal{S} \otimes \mathcal{T}$-measurable. Since $\{0\}$ is closed, $G r(f)$ is $\mathcal{S} \otimes \mathcal{T}$-measurable.

Marczewski's result is an immediate consequence of Theorem 4 and the following lemma, which follows from the facts that the product of two $(s)$-sets is an $(s)$-set in the product space while the class of $(s)$-sets form a $\sigma$-algebra. These facts can be found in [2]. From now on, we assume that $X$ and $Y$ are complete separable metric spaces.

Lemma 5. The class of $(s)$-measurable sets in a product space $X \times Y$ contains the smallest $\sigma$-algebra containing all the measurable rectangles $A \times B$ where $A$ is $(s)$-measurable in $X$ and $B$ is $(s)$-measurable in $Y$.

Corollary 6. Let $f: X \rightarrow Y$ be an (s)-measurable function where $X$ and $Y$ are complete separable metric spaces. Then the graph of $f, G r(f)$, is $(s)$ measurable in $X \times Y$. 


\section{References}

[1] C. Kuratowski, Topologie Vol. I, Monografie Matematyczne I II, Polish Academy of Sciences, Warszawa-lwow, 1933.

[2] E. Marczewski (Szpilrajn), Sur une classe de fonctions de M. Sierpinski et la classe correspondate d'ensembles, Fund. Math., 24 (1935), 17-34.

[3] E. Marczewski, Collected Mathematical Papers, Polish Academy of Sciences, Institute of Mathematics, Warszawa 1996. 